\title{
Fusion of Hematopoietic Cells with Purkinje Neurons Does Not Lead to Stable Heterokaryon Formation under Noninvasive Conditions
}

\author{
Christian Nern, ${ }^{1}$ Ines Wolff, ${ }^{1}$ Jadranka Macas, ${ }^{1}$ Josefine von Randow, ${ }^{1}$ Christian Scharenberg, ${ }^{2}$ Josef Priller, ${ }^{3}$ and \\ Stefan Momma ${ }^{1}$ \\ ${ }^{1}$ Institute of Neurology (Edinger Institute), Frankfurt University Medical School, D-60528 Frankfurt, Germany, ${ }^{2}$ Center for Experimental Hematology, \\ Department of Medicine, Karolinska University Hospital Huddinge, Karolinska Institute, S-14186 Stockholm, Sweden, and ${ }^{3}$ Neuropsychiatry and \\ Laboratory of Molecular Psychiatry CCM, Charité-Universitätsmedizin Berlin, D-10117 Berlin, Germany
}

Transplanted hematopoietic cells have previously been shown to contribute to cells of other tissues by cell fusion. We wanted to elucidate whether this phenomenon of cell fusion also occurs under physiological conditions. Using a transgenic mouse reporter system to irreversibly label cells of the hematopoietic lineage, we were able to test their contribution to other tissues in the absence of any additional and potentially confounding factors such as irradiation or chemoablation. We found genetically marked, fused Purkinje neurons as well as hepatocytes in numbers comparable to previous bone marrow transplantation studies. The number of fused Purkinje neurons increased after intrathecal administration of bacterial lipopolysaccharide, suggesting that cell fusion can be induced by inflammation. In contrast to previous studies, however, genetically labeled Purkinje neurons never contained more than one nucleus, and we found only a single cell containing two Y-chromosomes in a male mouse. Consistent with results from the mouse model and unlike human bone marrow transplant recipients, postmortem adult human cerebelli of nontransplanted individuals were devoid of binucleated or polyploid Purkinje neurons. Therefore, our data suggests that fusion of hematopoietic cells with Purkinje neurons is only transient and does not lead to stable heterokaryon formation under noninvasive conditions.

\section{Introduction}

Hematopoietic cells have been shown to be capable of adopting the phenotype and function of a variety of cell types of different organs in animal models as well as in humans (Herzog et al., 2003). Earlier reports explained this phenomenon with the high degree of plasticity of transplanted hematopoietic stem cells. However, although transdifferentiation of hematopoietic cells to cells of different tissues remains a possibility (Krause et al., 2001; Orlic et al., 2001; Wurmser et al., 2004), the principle mechanism of how bone marrow derived cells (BMDCs) adopt the phenotype of different organs appears to be via cell fusion (Terada et al., 2002; Ying et al., 2002; Wang et al., 2003). Two independent studies using bone marrow chimerism have provided compelling evidence for the fusion of BMDCs with cells of liver, muscle, and cerebellum in mice based on the detection of two nuclei in fused cells (Alvarez-Dolado et al., 2003; Weimann et al., 2003a). Furthermore, Johansson et al. (2008) show that fusion of BMDCs

\footnotetext{
Received Dec. 9, 2008; revised Jan. 22, 2009; accepted Feb. 16, 2009.

This work was supported by the Ludwig Edinger Foundation (to S.M.) and by the Deutsche Forschungsgemeinschaft and the Bundesministerium für Bildung und Forschung (to J.P.). We thank Dimitris Kioussis (National Institute for Medical Research, London) for generously providing the vav-iCre mice, Ruth von Laer and Thomas Fischer for help with mouse breeding, and Olaf Hoffmann for support with lumbar punctures.

Correspondence should be addressed to: Dr. Stefan Momma, Edinger Institute, Frankfurt University Medical

School, Heinrich-Hoffmann-Strasse 7, D-60528 Frankfurt, Germany. E-mail: stefan.momma@kgu.de.

I. Wolff's present address: Max Planck Institute for Developmental Biology, Tübingen, Germany.

D0I:10.1523/JNEUROSC1.5848-08.2009

Copyright $\odot 2009$ Society for Neuroscience $\quad$ 0270-6474/09/293799-09\$15.00/0
}

with Purkinje neurons of the cerebellum leads to reprogramming of the donor nucleus, resulting in the expression of Purkinje neuron $(\mathrm{PKN})$-specific molecules, underscoring a regenerative potential of fusion events. Indeed, there is evidence to suggest that transplantation of BMDCs derived from healthy animals can restore the function of non-hematopoietic organs, such as liver (Lagasse et al., 2000). Furthermore, the contribution of BMDCs to other organs, including the CNS, could be extended to humans (Mezey et al., 2003; Weimann et al., 2003b; Cogle et al., 2004; Crain et al., 2005). Examination of human postmortem brain specimens from females who had received sex-mismatched bone marrow transplantation after irradiation, revealed Y-chromosomecontaining PKNs in the cerebellum, which were polyploid, suggesting cell fusion (Weimann et al., 2003b). However, all of the above mentioned studies relied on highly invasive methods, such as irradiation to eliminate the host bone marrow and transplantation of donor bone marrow cells, to track the non-hematopoietic fate of hematopoietic cells. Therefore, we sought to address whether cell fusion (or transdifferentiation) can also occur under strictly physiological conditions, thus representing a possible endogenous mechanism for cell protection and regeneration by hematopoietic cells. To this end, we used a transgenic mouse model that combines the expression of a codon-improved Cre-recombinase (iCre) under the control of the hematopoietic-specific promoter vav, as described previously (de Boer et al., 2003), with a Cre-reporter mouse to irreversibly label cells of the hematopoietic lineage. This mouse model 
enabled us to determine the contribution of hematopoietic- to nonhematopoietic tissues under physiological conditions.

\section{Materials and Methods}

Mouse strains and genotyping. Transgenic vaviCre-mice have been described by de Boer et al. (2003) and were generously provided from D. Kioussis (National Institute for Medical Research, London, UK). These animals with originally mixed background were crossbred with C57BL/6 wild-type mice (Charles River Laboratories) and inbred for $>8$ generations. Primers used for genotyping: $5^{\prime}$-ctc tga cag atg cca gga ca and $5^{\prime}$-aca cca ttc ttt ctg acc cg. PCR-reactions using DNA of lysed mouse tails were performed under standard conditions including 40 cycles of amplification $\left(94^{\circ} \mathrm{C} 45 \mathrm{~s}, 60^{\circ} \mathrm{C} 30 \mathrm{~s}, 72^{\circ} \mathrm{C} 45 \mathrm{~s}\right)$. The PCR-product of 288 bp was visualized in a $1.5 \%$ agarose gel. Inbred vaviCre-mice with C57BL/6 background were either crossbred with floxed Rosa26-LacZ reporter mice (JAXmice stock number 003309) or with floxed Rosa26-EGFP reporter mice (JAX-mice stock number 004077). Genotyping was performed using the particular PCR-protocols provided by The Jackson Laboratory (http://www. jax.org). All animal experiments were performed according to the guidelines set by the Frankfurt University Medical School.

FACS analysis of peripheral blood. Peripheral blood was taken from the maxillary vein of adult animals and erythrocytes lysed by incubation with Erythrocyte lysis buffer (Roche Diagnostics) for $12 \mathrm{~min}$. Cells from vaviCre/GFP mice and controls (wild-type and reporter mice without vaviCre) were stained with the hematopoietic lineage marker CD45 (PerCP conjugated anti-mouse CD45, BD Biosciences PharMingen). Cells from vaviCre/LacZ mice and controls were fixed for $10 \mathrm{~min}$ in $4 \%$ PFA, permeabilized in PBS, $2 \%$ FCS, $0.1 \%$ Triton-X. Cells were stained with anti-CD45 and anti-LacZ antibodies (rabbit anti- $\beta$-Galactosidase, MP Biomedicals), washed and incubated with a secondary antibody against LacZ (Alexa Fluor488, goat- $\alpha$-rabbit IgG, Invitrogen). Analyses were performed with a FACS Canto II using the FACS Diva 6.11 Software (Becton Dickinson). Data analysis was done using FlowJo.

Cell transplantation and irradiation. BMDCs of donor mice were extracted by flushing femurs and tibias with HBSS supplemented with $2 \%$ FCS. After lysis of erythrocytes with lysis buffer, BMDCs were washed in PBS and kept on ice until further use. Recipient mice were lethally irradiated with a single dose of $9.5 \mathrm{~Gy}$. Three hours after irradiation, $\sim 5 \times$ $10^{6}$ BMDCs were injected into the retrobulbar venous plexus. After transplantation, mice were kept in filtertop-boxes and received antibiotic prophylaxis (Baytril) for $3 \mathrm{~d}$ subcutaneously. The dose for sublethal irradiation used in some experiments was $4.5 \mathrm{~Gy}$ (single dose).

Inflammation model. Brain inflammation was induced in transgenic mice by instillation into the lumbar spinal canal as described previously (Hoffmann et al., 2007) of $1 \mu \mathrm{g}$ of LPS 026:B6 (Sigma) dissolved in $40 \mu \mathrm{l}$ of PBS. Intrathecal administration of $1 \mu \mathrm{g}$ of LPS was repeated after 2 weeks. Adequate recovery from anesthesia and absence of pareses were verified after each administration.

Human specimens. Autopsied human brains were obtained within $24 \mathrm{~h}$ of death from the Department of Pathology of the University Hospital Frankfurt or as tissue blocks embedded in paraffin from the Edinger Institute archive. We used tissue that was not needed for further diagnostic purpose, which was taken from sagittally cut cerebellar hemispheres and vermis (3-5-mm-thick slices). Tissue that was to be used for fluorescence in situ hybridization (FISH) was stored in ice-cold PBS and then snap frozen in methylbutane, cooled with liquid nitrogen. Tissue for light and confocal microscopic screening and electron microscopy was fixed in paraformaldehyde ( $4 \%, \mathrm{pH} 7.4)$ at $4^{\circ} \mathrm{C}$ for at least $24 \mathrm{~h}$ and then shifted to PBS with $15 \%(\mathrm{w} / \mathrm{v})$ sucrose or embedded in paraffin. For further staining-procedures, the tissue was cryosectioned into 5-12 $\mu \mathrm{m}$ slices. All work involving human tissue was approved by the ethical committee of the Frankfurt University Medical School.

Tissue preparation. Before organ-extraction, deeply anesthetized mice were transcardially perfused with $50 \mathrm{ml}$ of PBS followed by perfusion with $50 \mathrm{ml}$ of ice-cold paraformaldehyde (4\%, pH7.4). Organs were postfixed overnight in $4 \%$ paraformaldehyde and cryo-protected with $20 \%$ sucrose for $24 \mathrm{~h}$. For quantitative immunohistochemical and B-gal staining, the tissue was snap frozen in methylbutane cooled in liquid nitrogen. Serial 12-15 $\mu \mathrm{m}$ cryostat (SM2000R, Leica, Germany) sections were cut onto glass slides and stored at $-20^{\circ} \mathrm{C}$ until further processing. For EM- and CLSM-analysis, tissue was cut into serial $40-60 \mu \mathrm{m} \mathrm{sec-}$ tions on a Vibratome (VT 1000S, Leica) and stored at $4^{\circ} \mathrm{C}$ in PBS before further processing.

Staining procedures and microscopy. For enzymatic $\beta$-gal-staining, tissue-slices were first permeabilized for $30 \mathrm{~min}$ in phosphate buffer containing NP-40 (0.02\%), potassiumhexacyano-ferrat $(5 \mathrm{~mm})$ and -ferrit (5 mM), sodiumdeoxycholat $(0.25 \mathrm{~mm})$, and magnesium chloride $(2 \mathrm{~mm})$ and subsequently incubated at $37^{\circ} \mathrm{C}$ overnight after addition of $\beta$-gal substrate solution (1:20) containing $\beta$-galactopyranoside solved in dimethylformamid $(20 \mathrm{mg} / \mathrm{ml})$.

For immunohistochemical and immunofluorescent antibodystaining, the following primary antibodies (ABs) were used: anti- $\beta$ galactosidase (mouse monoclonal, Promega) anti-CD11b (mouse monoclonal, BD PharMingen), anti-Albumin (rabbit polyclonal, Accurate), anti-Calbindin (mouse monoclonal, Sigma), and anti-GFP-Alexa 488 conjugated (rabbit polyclonal, Invitrogen). Subsequently, the following secondary ABs were used: Alexa Fluor488 (goat- $\alpha$-mouse IgG, goat- $\alpha$-rabbit IgG, Invitrogen) and Alexa Fluor568 (goat- $\alpha$-mouse IgG, goat- $\alpha$-rabbit IgG, Invitrogen). For peroxidase immunohistochemistry, a diaminobenzidine-reaction or the StrAviGen Multilink-RKit (BioGenex) with 3-amino-9-ethyl-carbazole as a chromogen (AEC; BioGenex) was applied. Nuclear staining was performed with DAPI (300 $\mathrm{mM}$, Invitrogen), Propidiumiodide (Invitrogen), Toto-R-3-iodide (Mo$\mathrm{BiTec}$ ), or cytox green (Invitrogen). Images for light microscopy were taken on a BX51 microscope (Olympus) using the Analysis Software package (Soft Image Systems). Fluorescence images were analyzed and recorded on a Leica TCS SP confocal microscope, using the EZ-C1 software or on a Nikon TE2000-E using the EC-C1 3.60 Software. After recording, digital images were further processed and adjusted for brightness, contrast and color balance with Image $(\mathrm{NIH})$. Final figures were assembled in Adobe Photoshop CS2 Version 9.0.

Electron microscopic analysis. $50 \mu \mathrm{m}$ Vibratome-sections of vav-iCre/ LacZ mice with enzymatically visualized LacZ-expressing cells were postfixed in $2 \%$ glutaraldehyde, dehydrated, and embedded in epoxy resin. Epoxy resin blocks were trimmed to minimal size with the labeled cell at the center. Ultrathin sections (50 nm; $\sim 200$ per LacZ-expressing cell) were serially cut on a Leica Ultracut UCT, stained with uranyl acetate and lead citrate, and analyzed using a Zeiss EM 10CR TEM. Images were recorded using the Gatan BioScan camera. For ultrastructural analysis of human Purkinje neurons, $3 \mu \mathrm{M}$ paraffin sagittal sections were postfixed in $2 \%$ glutaraldehyde, dehydrated, re-embedded in epoxy resin, and $\sim 60$ ultrathin sections $(50 \mathrm{~nm}$ ) were cut serially on a Leica Ultracut UCT.

Fluorescence in situ hybridization. For murine Y-FISH-staining procedures, animals were perfused with PBS, cerebelli dissected out and snap frozen in methylbutan. Serial $8 \mu \mathrm{m}$ sections were cut and postfixed for 2 min in 2\%PFA. In situ hybridization was performed using the starFISHkit (Cambio) following the manufacturers instructions. FISH on human tissue was performed using X-chromosome probes from Vysis (VysisAbbott), following manufacturers instructions.

Quantification and statistical analysis. For quantification of labeled $\mathrm{PKN}$ in the mouse cerebellum, whole cerebelli were serially cut and LacZ- or GFP-positive PKNs were counted from $1 / 4$ of all sections. Values are expressed as means $\pm \mathrm{SD}$. A two-tailed Student's $t$ test, assuming the same variances was performed for statistical analysis of the data using the BiAS Software (Frankfurt University).

\section{Results}

Detection of hematopoietic cell contribution to nonhematopoietic tissues with the vav-iCre/LacZ transgenic reporter system

To detect the contribution of hematopoietic cells to nonhematopoietic tissues without any additional manipulation, we needed to irreversibly label endogenous hematopoietic cells accounting for the fact that these cells may lose all hematopoietic markers after cell fusion or transdifferentiation. To achieve this, transgenic mice that express iCre under the control of the hematopoietic cell-specific vav-promotor (de Boer et al., 2003) were 
a
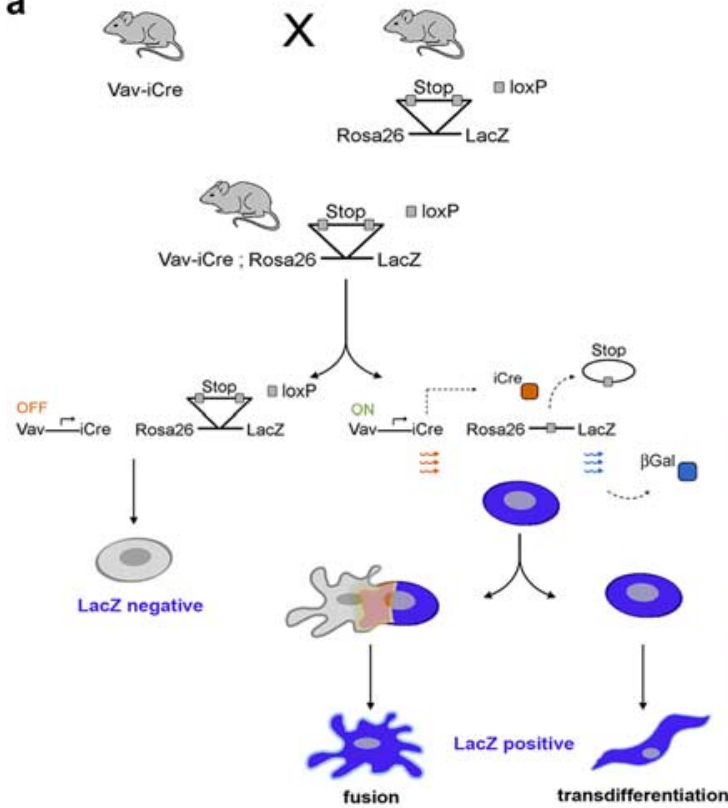

b

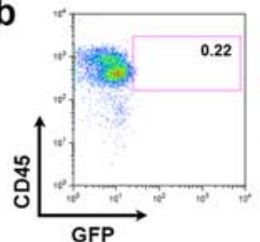

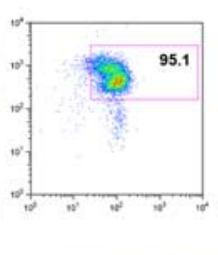

C
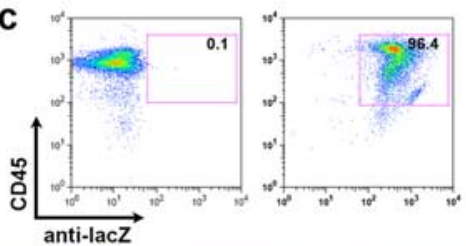
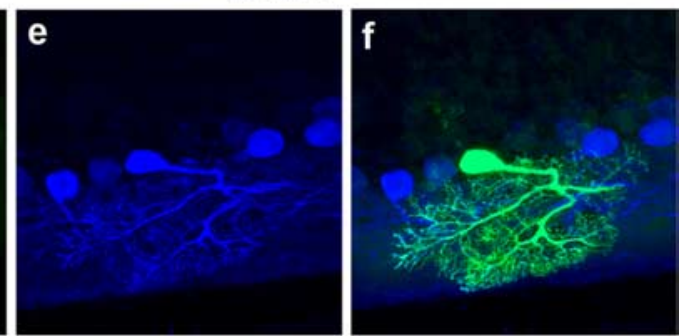
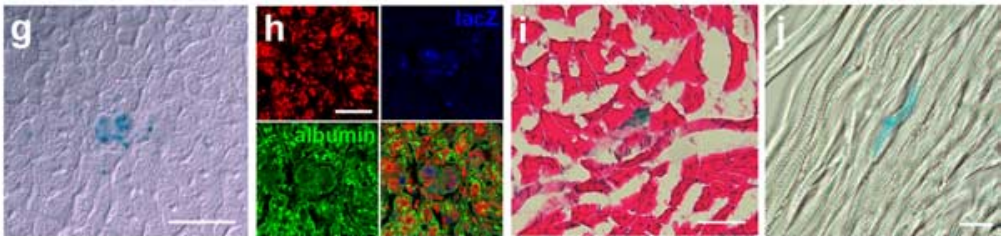

Figure 1. The vav-iCre/LacZ reporter mouse reveals the contribution of hematopoietic cells to non-hematopoietic tissues. $\boldsymbol{a}$, iCre is expressed under the control of the hematopoietic-specific vav-promoter in vav-iCre mice. Crossing vav-iCre mice with Cre reporter lines leads to the excision of a floxed stop codon in hematopoietic cells and subsequently to the expression of LacZ under the control of the lineage-independent ROSA26 promoter. Thus, reporter gene expression is maintained, irrespectively of changes in lineage-specific genes. $\boldsymbol{b}$, FACS-analysis of mononucleated cells from peripheral blood of adult vaviCre/GFP mice for recombination frequency in CD45-positive cells. Fluorescence intensity for CD45-stain is indicated on the $y$-axis, for GFP, on the $x$-axis. The left panel shows a control mouse. On the right panel the analysis of peripheral blood from a double transgenic mouse is shown. High fluorescence intensity is observed in $>95 \%$ of the cells. $\boldsymbol{c}$, As in $\boldsymbol{b}$, but showing the analysis for vaviCre/LacZ mice. Intracellular LacZ was stained by an antibody in fixed and permeabilized cells. The recombination frequency is $>96 \%$. $\boldsymbol{d}$, Identification of a GFP-positive cell in the cerebellum of a vav-iCre/GFP reporter mouse, displaying the typical highly arborized morphology of a Purkinje neuron. $\boldsymbol{e}$, Immunoreactivity for the Purkinje neuron specific marker calbindin (blue) and overlay of GFP and calbindin images ( $\boldsymbol{f}$ ). $\boldsymbol{g}$, Identification of a LacZ-positive cell by X-gal enzymatic staining in the liver of a vav-iCre/LacZ reporter mouse. Confocal image of the same cell with (h) propidium iodide (red), LacZ-stain (blue), and albumin (green), overlay. $\boldsymbol{i}$, Skeletal muscle with a LacZ-positive muscle fiber (blue), counterstained with hemalaun and eosin, and a LacZ positive cardiac muscle fiber in $\boldsymbol{j}$. Scale bars: $\boldsymbol{d}, 20 \mu \mathrm{m} ; \boldsymbol{g}, 10 \mu \mathrm{m} ; \boldsymbol{h}, 5 \mu \mathrm{m} ; \boldsymbol{i}, \boldsymbol{j}, 25 \mu \mathrm{m}$.

crossbred with mice that express as reporter gene $\beta$-galactosidase (LacZ), green fluorescent protein (GFP), or yellow fluorescent protein (YFP) under the control of the ROSA26 promoter. After iCre-mediated enzymatic excision of a floxed stop-codon, the marker protein is expressed and hence the mice are called vaviCre/LacZ (-GFP, -YFP) mice throughout this manuscript. The vav gene codes for a protein, which is involved in various signaling processes and is expressed exclusively in hematopoietic cells (Georgiades et al., 2002; de Boer et al., 2003). Therefore, in our model, LacZ-expression should be observed only in hematopoietic cells, transdifferentiated hematopoietic cells, or cells that underwent fusion with hematopoietic cells (a schematic description of the model is given in Figure 1a). To assess recombination frequency in hematopoietic cells of our reporter mice, we performed a fluorescence-activated cell sorting (FACS) analysis of peripheral blood of double transgenic mice (vaviCre/GFP and -LacZ) to quantify hematopoietic CD45-positive cells expressing GFP (Fig. 1b) or LacZ (Fig. 1c) respectively. Our analysis shows that $>95 \%$ of all CD45-positive cells carry the marker gene.

Next, we analyzed LacZ- and/or GFP-expression in liver, heart, skeletal muscle, and brain in vav-iCre/LacZ (-GFP) mice aged 2 months to 12 months. Consistent with previous studies using bone marrow chimeras, we could detect cells expressing the reporter gene in liver and brain of all mice analyzed. In the brain, expression of the reporter gene was restricted to Purkinje neurons (Fig. 1d), characterized by their distinct, highly arborized morphology and the expression of calbindin (Fig. 1e,f). LacZpositive cells in the liver (Fig. $1 g$ ) were identified as hepatocytes via additional immunostaining against albumin (Fig. 1h). Expression of LacZ was detected by enzymatic 5-bromo-4-chloro- 3-indolyl- $\beta$-D-galactopyranoside (X-gal) and confirmed by antibody staining. The results were confirmed using reporter mice expressing GFP or YFP instead of LacZ. Screening of reporter mice or vav-iCre mice alone never showed any LacZ-/GFP-/YFPpositive cells in the brain, liver or any other organ. Quantification of LacZ-expressing Purkinje neurons and hepatocytes in vaviCre/LacZ mice showed numbers of potentially fused cells comparable to those found in previous bone marrow transplantationstudies, i.e., $28 \pm 15 \mathrm{SD}(n=6)$ LacZ-positive PKNs per $1 / 2$ cerebellum in animals 2 months of age and $23 \pm 27$ SD LacZpositive hepatocytes per $1 \times 10^{6}$ cells in the liver. LacZ-positive skeletal muscle cells (Fig. 1i) occurred very rarely and only in two of the six mice that were analyzed by screening $>5 \mathrm{~cm}^{2}$ of tissue sections from the anterior tibial muscles of each mouse. Likewise, we were only able to detect a single LacZ-positive muscle fiber in the cardiac muscle of one mouse $(n=4)$ (Fig. $1 j)$. These results demonstrate that the contribution of hematopoietic cells to nonhematopoietic tissues can be analyzed with the vav-iCre/reporter mouse model in non-irradiated healthy transgenic mice. The distribution and frequency of potentially fused PKNs and hepatocytes is comparable to that found in hematopoietic chimerism models.

\section{Labeled Purkinje neurons in the vav-iCre/LacZ mouse model} are not binucleated

Cells from muscle and liver can contain multiple nuclei in contrast to neurons, which usually just contain one nucleus. Therefore, the occurrence of binucleated PKNs expressing the marker gene of transplanted donor hematopoietic cells in irradiated bone marrow chimeras led to the conclusion that acquisition of the 
genetic marker was caused by cell fusion rather than transdifferentiation (AlvarezDolado et al., 2003). To examine whether, in our model, LacZ-expression was also acquired by cell fusion, LacZ-positive PKNs were analyzed in more detail. Surprisingly, none of the $>80 \mathrm{PKN}$ analyzed by three-dimensional (3D) confocal laser scanning microscopy (CLSM) were binucleated (Fig. 2a-f). To exclude technical limitations in the detection of binucleated cells, we transplanted bone marrow cells from $\beta$-actin GFP transgenic mice or from vav-iCre/LacZ mice into lethally irradiated wild-type hosts. Consistent with previously published results, we found binucleated PKNs $>6$ months after transplantation (Fig. $2 g-l$ ), thereby excluding the possibility of shortcomings in our detection techniques. To confirm these results, Vibratome sections obtained from the cerebellum of vav-iCre/LacZ mice with LacZ-positive PKNs $(n=5)$ (Fig. $3 a, b)$ were re-embedded in epoxy resin and cut into serial ultrathin sections. Purkinje neurons are characterized by their specific location between the molecular and granule cell layer and beside their distinct dendrite morphology have very large cell bodies. LacZ positive cells were almost always located in this layer and had a large cell body. The few exceptions were cells associated with blood vessels and of much smaller size (data not shown). Because $\mathrm{X}$-gal distribution does only sparsely occur throughout thin dendrites, we confirmed the Purkinje neuron identity of X-galpositive cells by counterstaining with calbindin in additional sections. PKN identity could be confirmed without exceptions $(n=15)$ (Fig. $3 c)$. Examination by electron microscopy (EM) confirmed that the previously X-gal-stained PKNs had only one nucleus (Fig. $3 d-g$ ). One obvious difference to the experimental procedure of previous studies was irradiation with a lethal dose. We made an attempt to establish whether irradiation could influence the nuclear phenotype of PKNs. vaviCre/LacZ mice older than 6 months were sublethally irradiated with a single dose of 4.5 Gy. After another 6 months, Purkinje neurons in these animals were analyzed for the number of nuclei. CLSM-analysis of LacZ-expressing PKNs $(n=15)$ in sublethally irradiated vaviCre/LacZ mice exhibited no difference to non-irradiated animals in that only one nucleus could be detected in LacZexpressing PKNs (data not shown).

\section{LacZ-positive Purkinje neurons in male vav-iCre/LacZ mice} do not contain supernumerous Y-chromosomes

One possible explanation for the occurrence of LacZ-positive PKNs carrying only one nucleus could be that, under physiolog-
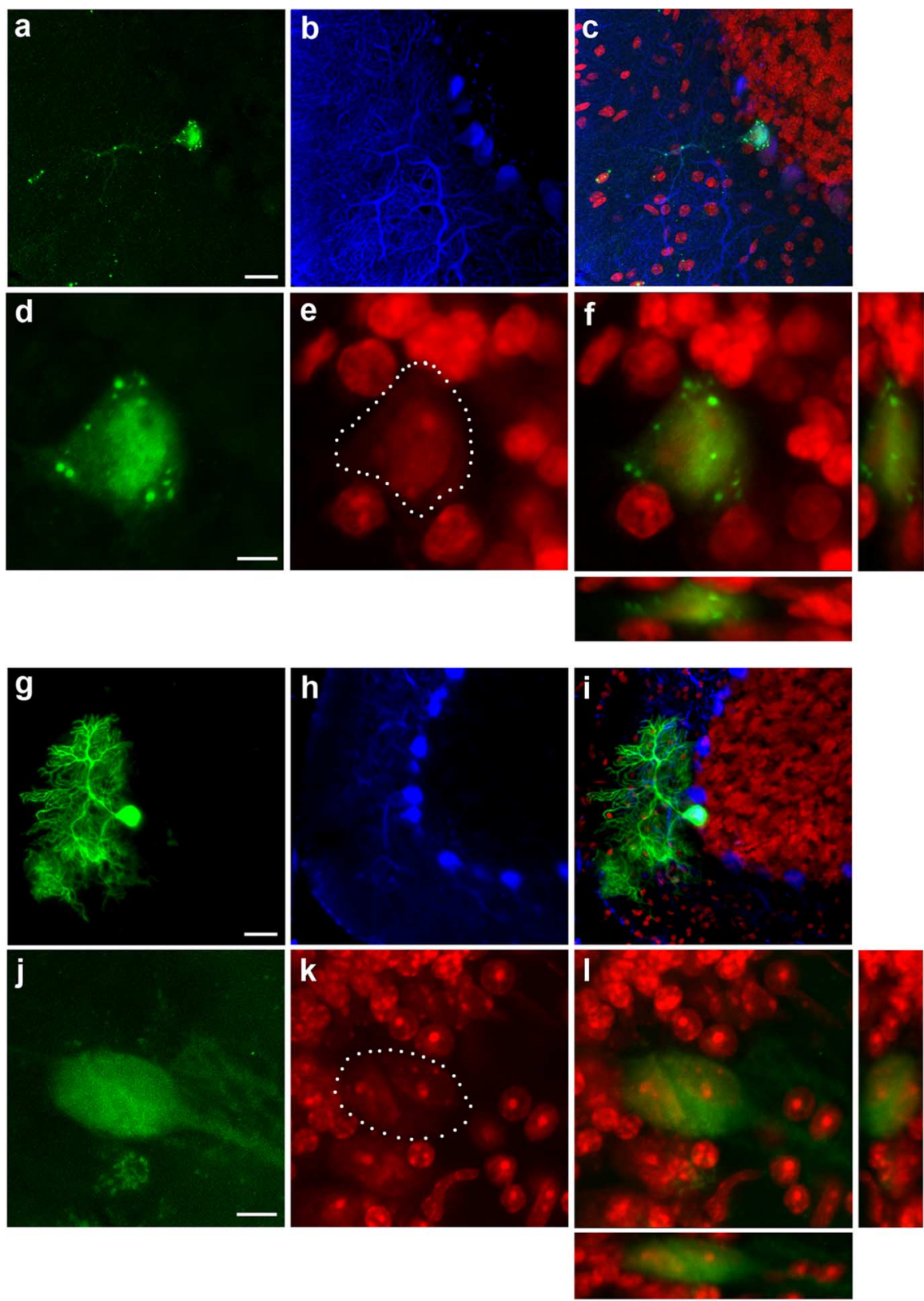

Figure 2. LacZ-labeled Purkinje neurons in vav-iCre/LacZ mice are not binucleated in contrast to PKNs in irradiated bone marrow chimeras. $\boldsymbol{a}$, LacZ-positive PKN in the cerebellum visualized by immunostaining in a vav-iCre/LacZ mouse. Long dendritic processes can be seen with typical punctuate staining pattern of bacterial LacZ. $\boldsymbol{b}$, Calbindin stain of the same area and in (c) overlay of $\boldsymbol{a}$ and $\boldsymbol{b}$ together with nuclear PI-counterstain in red. $\boldsymbol{d}$, Magnification of the same cell showing LacZ immunoreactivity; $\boldsymbol{e}$, Pl-counterstaining showing a single large nucleus in the labeled PKN with the cell body outlined by white dots and in $(\boldsymbol{f})$ overlay of the two images with panels showing 3D-projections through the $x$ - and $y$-axis. $\boldsymbol{g}$, GFP-positive Purkinje neuron in a wild-type mouse that received a bone marrow transplant of actin-GFP reporter mice 12 months earlier. $\boldsymbol{h}$, Calbindin stain of the same area and in (i) overlay of the images plus PI-counterstain in red. $\boldsymbol{j}$, Another GFP-positive Purkinje neuron from the same animal. $\boldsymbol{k}$, PI-stained nuclei with the cell body outlined by white dots, showing two nuclei in the same cell and overlay in / with $3 \mathrm{D}$ projections through $x$ - and $y$-axis. Scale bars: $\boldsymbol{a}, \boldsymbol{g}, 20 \mu \mathrm{m} ; \boldsymbol{d}, \boldsymbol{j}, 5 \mu \mathrm{m}$.

ical conditions, fusion of hematopoietic cells with PKNs in vav$\mathrm{iCre} / \mathrm{LacZ}$ mice is followed by nuclear fusion. This has been reported to occur in rare instances between mesenchymal stem cells and small airway epithelial cells (Spees et al., 2003). To test this hypothesis, we analyzed LacZ-positive PKNs from male vaviCre/LacZ mice for the number of Y-chromosomes using FISH (Fig. 4). Analysis of a total of 78 LacZ-positive PKNs from two mouse brains aged 7 and 8 months, respectively, yielded nine cells containing no Y-chromosome, 68 cells with one (Fig. 4a), and 


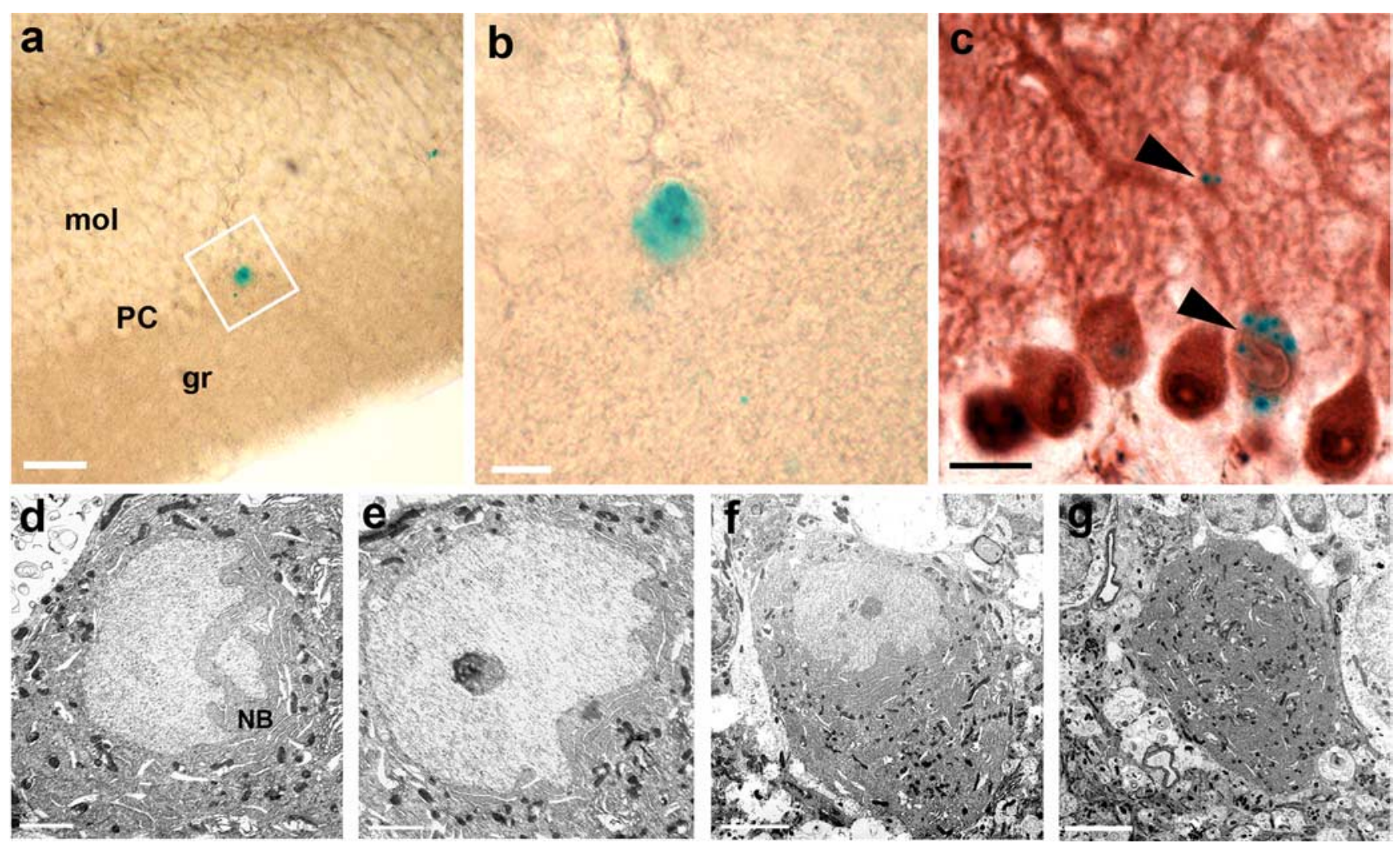

Figure 3. Electron microscopic confirmation of single nuclei in LacZ-labeled Purkinje neurons in vav-iCre/LacZ mice. $\boldsymbol{a}$, Example of a LacZ-positive Purkinje neuron, detected by X-gal staining of a 50- $\mu \mathrm{m}$-thick Vibratome section, located in the Purkinje neuron layer (PC), between molecular ( $\mathrm{mol}$ ) and granule ( $\mathrm{gr}$ ) cell layer. $\boldsymbol{b}$, Larger image of the same cell as in $\boldsymbol{a}$. $\boldsymbol{c}$, Light microscopic staining of calbindin confirming that all large X-gal-positive cell bodies in the PC are indeed Purkinje neurons. Typical dotted X-gal stain in the cell body and dendrite indicated by black arrowheads. $\boldsymbol{d}$, $\boldsymbol{e}$, Serial ultrathin sections through the same PKN as shown in $\boldsymbol{a}$ and $\boldsymbol{b}$ reveal only a single nucleus. The nucleus is large and light with a dark basophilic nucleolus, with deep folds and invaginations toward the side of the dendrites and Nissl bodies (NB) at the side of the folds, thus displaying the typical morphologic features of Purkinje neurons. $\boldsymbol{f}, \boldsymbol{g}$, Same cell at different $z$-levels and smaller magnification showing no second nucleus in this neuron. Scale bars: $\boldsymbol{a}-\boldsymbol{c}, 20 \mu \mathrm{m} ; \boldsymbol{d}, \boldsymbol{e}, 2 \mu \mathrm{m} ; \boldsymbol{f}, \boldsymbol{g}, 5 \mu \mathrm{m}$.
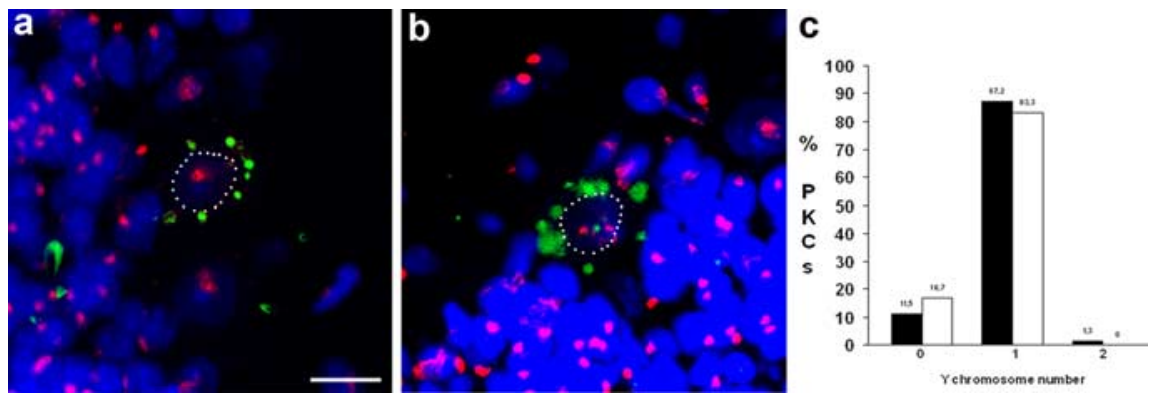

Figure 4. Number of Y-chromosomes in mononucleated LacZ-positive Purkinje neurons in vav-iCre/LacZ mice. $\boldsymbol{a}$, LacZ-positive PKN containing one Y-chromosome, detected by FISH (red). Nuclear counterstain cytox green (blue), LacZ recorded in transmission light, inverted, and overlayed (green). $\boldsymbol{b}$, PKN containing two $Y$-chromosomes. Nuclei in $\boldsymbol{a}$ and $\boldsymbol{b}$ are outlined by white dots. c, Quantification of number of Y-chromosomes in LacZ-positive (black bars) and negative (white bars) PKNs. Scale bar: $\boldsymbol{a}, \boldsymbol{b}$ (in $\boldsymbol{a}$ ) $20 \mu \mathrm{m}$.

one single cell with two Y-chromosomes (Fig. 4b), suggesting that nuclear fusion is rare. For a better assessment, we also quantified the number of Y-chromosomes in unlabeled PKNs $(n=118)$ (Fig. 4c). We found that $17 \%$ of LacZ-negative PKNs contained no Y-chromosome signal compared with $12 \%$ in LacZ-positive PKNs. Moreover, $83 \%$ of LacZ-negative PKNs contained one Y-chromosome signal compared with $87 \%$ in LacZ-positive PKNs. The detection of only one LacZ-positive PKN containing two Y-chromosomes and the absence of significant differences in the number of Y-chromosomes between labeled and unlabeled Purkinje neurons argues against nuclear fusion as the predominant mechanism involved in the occurrence of mono- nucleated LacZ-positive Purkinje neurons in vav-iCre/LacZ mice.

Inflammation increases the frequency of cell fusion events between hematopoietic cells and cerebellar Purkinje neurons without generation of binucleated cells

Pathological processes were found to increase the contribution of hematopoietic cells to liver and muscle (Corbel et al., 2003; Willenbring et al., 2004). To determine whether pathological changes in the brain also enhance the fusion of hematopoietic cells with PKNs, we administered bacterial lipopolysaccharide (LPS) intrathecally to vav-iCre/LacZ mice. LPS is known to induce an inflammatory reaction in the brain, and we found an increased number of CD11b-positive microglia in the cerebellum at 2-3 months after LPS administration (Fig. $5 a, b$ ). Quantification of LacZ-expressing PKNs showed a mean number of $55 \pm 12$ per $1 / 2$ cerebellum in LPS-treated transgenic mice $(n=4)$ compared with $37 \pm 14$ in untreated transgenic animals of matched age ( $n=4$, Student's $t$ test, $p=0.045)$. Interestingly, all of the LacZpositive PKNs in LPS-treated vav-iCre/LacZ mice were mononucleated (Fig. $5 c-e$ ), suggesting that the binucleated Purkinje neurons found in bone marrow chimeras are not simply the result of irradiation-induced inflammatory changes. 

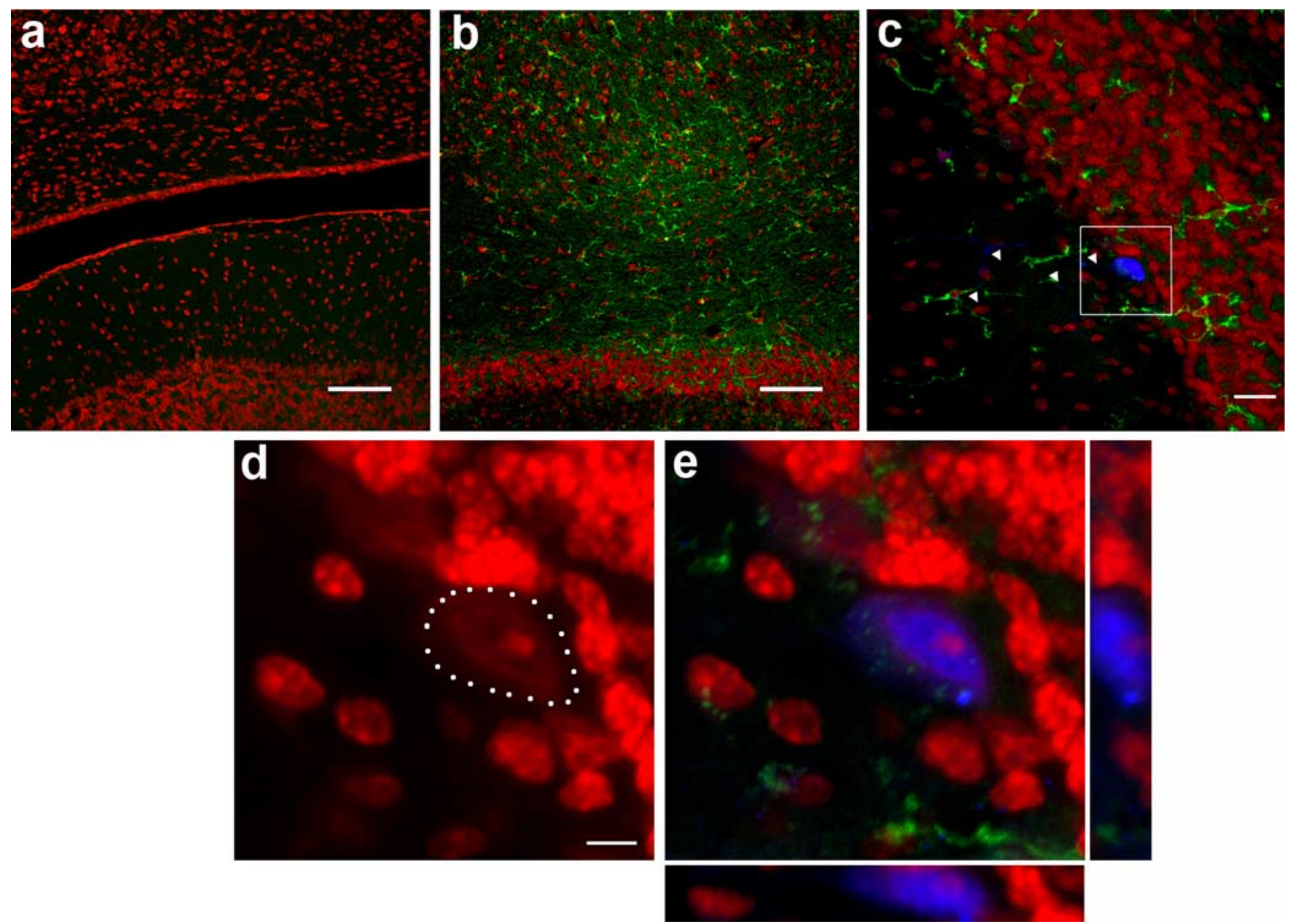

Figure 5. Inflammation increases the frequency of cell fusion events between hematopoietic cells and Purkinje neurons. $\boldsymbol{a}, \boldsymbol{b}$, Staining with the marker CD11b (green) and counterstain of nuclei with PI (red) in control animals (a) and 2-3 months after injection of LPS shows inflammatory reaction (b). c, Overview of a LacZ-positive Purkinje neuron (blue) with long dendrites (indicated by white arrowheads) and (D11b-positive cells (green) distributed in the molecular and granule cell layer. $\boldsymbol{d}$, Enlargement of the same cell, showing a single nucleus counterstained with PI and the cell body outlined by white dots. $\boldsymbol{e}, 3 \mathrm{D}$ view in combination with calbindin and CD11b. Scale bars: $\boldsymbol{a}, \boldsymbol{b}, 100 \mu \mathrm{m} ; \boldsymbol{c}, 20 \mu \mathrm{m} ; \boldsymbol{d}, 5 \mu \mathrm{m}$.

\section{No evidence for binucleated Purkinje neurons in the normal adult human cerebellum}

Fusion of hematopoietic cells with PKNs resulting in binucleated PKNs was found to occur in patients, who had received total body irradiation followed by bone marrow transplantation (Weimann et al., 2003b). To determine whether binucleated PKNs are also present in the human brain under physiological conditions, cerebellar tissue from postmortem brains of patients without neuropathological changes $(n=5)$ (Table 1$)$ was screened for binucleated PKNs. A large number of serial sections $(n=100)$ were cut from each cerebellum and counterstained with hematoxylin/eosin. Between 80,000 and 120,000 Purkinje neurons were analyzed in each of the five brains by light microscopy. Cells that appeared binucleated (Fig. $6 a$ ) were selected and subsequently analyzed ultrastructurally in serial ultrathin sections. After careful evaluation, none of the preselected cells was found to be binucleated (Fig. $6 b$ ), which is in line with the observations made in vav-iCre/LacZ mice. Among $>500,000$ screened PKNs in hu-

\begin{tabular}{llllll}
\multicolumn{6}{l}{ Table 1. List of human brains analyzed for binucleated Purkinje neurons } \\
\hline Number & Age (years) & Sex & Analysis & Pathologies & Cause of death \\
\hline 1 & 63 & $\mathrm{~m}$ & FISH & & Myocardial infarction \\
2 & 53 & $\mathrm{f}$ & FISH & Liver cirrhosis, ovarian tumor & \\
3 & 82 & $\mathrm{~m}$ & FISH & & Myocardial infarction \\
4 & 83 & $\mathrm{~m}$ & FISH & Malign melanoma & \\
5 & 63 & $\mathrm{f}$ & FISH & AML & \\
6 & 63 & $\mathrm{f}$ & FISH & AML & Myocardial infarction \\
7 & 63 & $\mathrm{~m}$ & Histology & & \\
8 & 63 & $\mathrm{~m}$ & Histology & Sepsis & Stabbing \\
9 & 29 & $\mathrm{~m}$ & Histology & AlDS, hepatitis C & \\
10 & 50 & $\mathrm{~m}$ & Histology & Alcoholic, depression & \\
11 & 57 & $\mathrm{~m}$ & Histology & Alcoholic, liver cirrhosis & \\
\hline
\end{tabular}

m, Male; f, female; AML, acute myeloid leukemia. man brains, only a single PKN contained two nuclei (Fig. $6 c$ ). The fact that these nuclei have an identical morphology, typical for PKNs, suggests that either it was not contributed by a hematopoietic cell, or that cell fusion occurred a long time ago, with subsequent reprogramming and changes in morphology. Moreover, analysis of $\sim 1000$ PKNs from each of six additional brains (Table 1) for aberrant X-chromosome numbers after visualization by FISH (Fig. $6 d$ ) revealed no PKN containing more than a diploid set of sex chromosomes. 


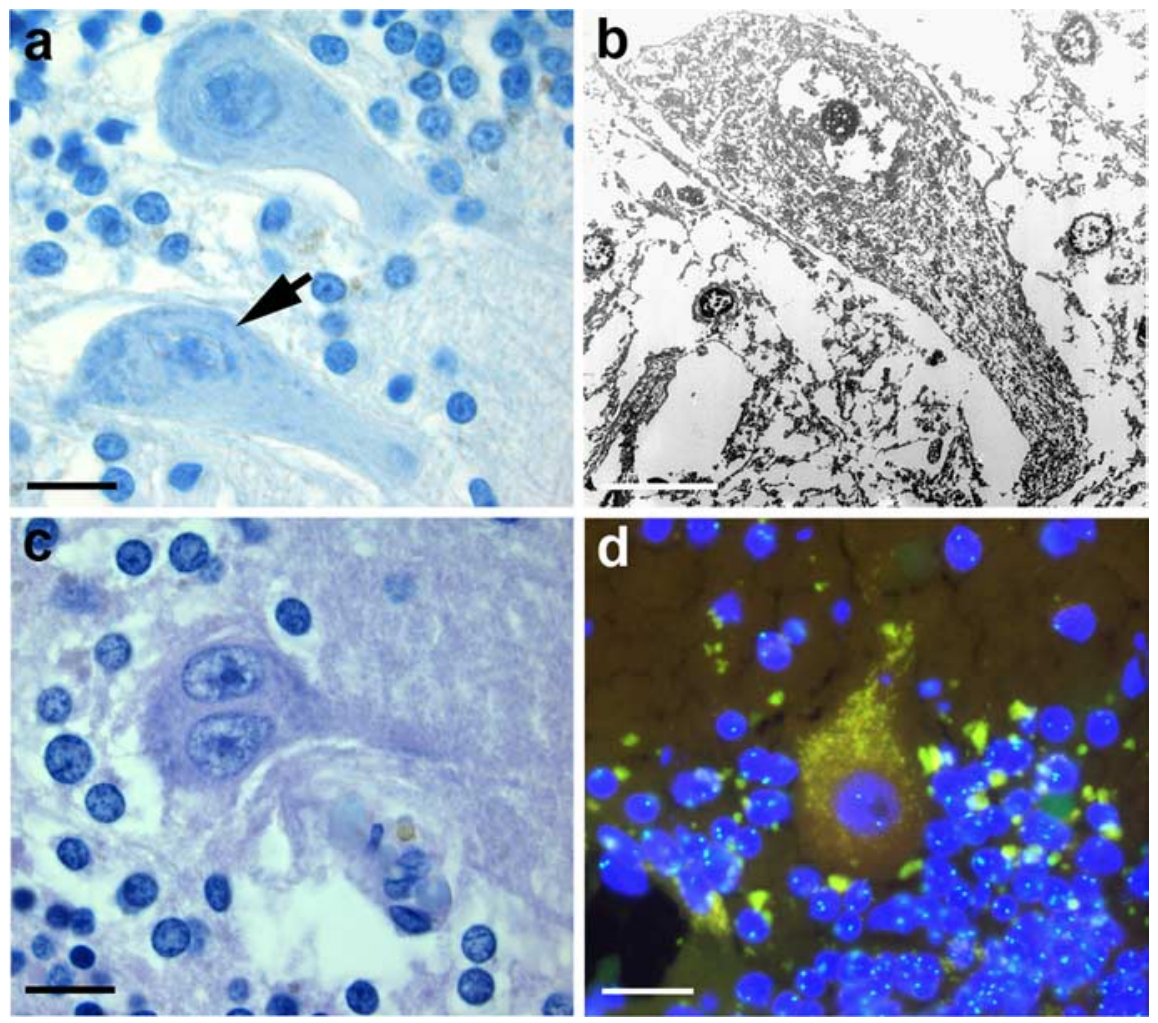

Figure 6. Purkinje neurons in the adult human brain are mononucleated. $\boldsymbol{a}$, PKN with morphological appearance of two heterogenous nuclei indicated by black arrow. $\boldsymbol{b}$, Same PKN as indicated in $\boldsymbol{a}$, re-embedded and processed for electron microscopy shows only one nucleus. c, Sole exception of a PKN clearly showing two nuclei, although with identical morphology. $\boldsymbol{d}$, Using FISH to screen for X-chromosomes never yielded any cell with more than a diploid chromosome number. Scale bars: $\boldsymbol{a}-\boldsymbol{d}, 10 \mu \mathrm{m}$.

described by Georgiades et al. (2002), who showed that vav-iCre-mediated recombination was specific for cells of the hematopoietic compartment. Georgiades et al. also found labeling of the vascular endothelium, which was not observed in any of the other vav-iCre mouse lines published. A more recent study using vav-iCre/YFPreporter mice to address the question whether hematopoietic cells contribute to liver cells during development and adulthood found $\sim 2$ YFP-expressing cells per 100,000 liver cells (Stadtfeld and Graf, 2005). Although the number of LacZexpressing hepatocytes varied considerably between individuals in our study, we found on average 2.3 LacZ-expressing cells per 100,000 hepatocytes, which is consistent with the findings of Stadtfeld and Graf (2005). The detection of reportergenepositive non-hematopoietic cells has been explained by the fusion of hematopoietic cells with cells from the tissues analyzed or by transdifferentiation of hematopoietic cells. In the case of fusion, the occurrence of two nuclei resulting from stable heterokaryon formation has been suggested (Weimann et al., 2003a). Surprisingly, our results show that potentially fused LacZpositive PKNs in healthy non-irradiated transgenic mice are mononucleated. We confirmed the presence of binucleated Purkinje neurons in lethally irradiated

\section{Discussion}

The ability of BMDCs to differentiate into cells of different organs has been discussed controversially within the last years. After initial reports about the transdifferentiation of BMDCs into nonhematopoietic cell types in vitro and in vivo, cell fusion has been suggested as the main mechanism behind the observed plasticity of BMDCs (Alvarez-Dolado, 2007). Endogenous cell fusion has been discussed in different contexts, including physiological mechanisms like spermatozoa-ovum fusion, developmental processes like the formation of placenta, bone, and muscle (Potgens et al., 2002; Horsley and Pavlath, 2004; Chen et al., 2007), but also in the context of pathological processes like virus-induced fusion (Price et al., 1988) and tumorigenesis (Bjerkvig et al., 2005). All previous studies dealing with the investigation of fusion events in adult animals have used experimental procedures such as cell transplantation after lethal irradiation, transplantation of cells from syngeneic mice, or parabiotic models to generate chimerism (Wagers et al., 2002; Massengale et al., 2005; Magrassi et al., 2007). However, although these approaches differ significantly in the way they impact the animal, none of these approaches is physiological in a strict sense and therefore limited in establishing the contribution of endogenous BMDCs to other organs. In this study, we determined the contribution of endogenous hematopoietic cells to Purkinje neurons in healthy non-irradiated transgenic animals. We used the vav-iCre transgenic mouse model established by de Boer et al. (2003) in combination with the ROSA26-LacZ (-GFP, -YFP) Cre-reporter mice to irreversibly shift reporter gene expression under the control of the ROSA26promoter, thus making it independent of lineage-specific expression. The vav-iCre transgenic mouse model has previously been wild-type mice transplanted with bone marrow from GFP transgenic mice as described previously (Priller et al., 2001), or with bone marrow from vav-iCre/LacZ mice. Thus, the difference between our observations and previous findings cannot be explained only by the different experimental conditions used, e.g., animal housing, genetic markers, detection methods, and the like. We considered five explanations for the occurrence of mononucleated LacZ-positive Purkinje neurons in vav-iCre/ LacZ transgenic mice: First, hematopoietic cells have transdifferentiated, as originally suggested; second, unspecific expression of Cre recombinase in Purkinje neurons; third, cell fusion occurred with concomitant nuclear fusion; fourth, cells fused with or without nuclear fusion, but genetic material from the donor cells was lost rapidly; and fifth, transfer of Cre-protein or cre-messenger RNA occurred via transient fusion or intercellular transport by vesicles, thus causing the genomic excision of the stop cassette leading to reporter gene expression.

Although transdifferentiation remains a formal possibility, we consider it unlikely given that we never saw anything that could amount to intermediate stages of a differentiation process leading from a hematopoietic cell to a Purkinje neuron. Leaky Cre expression is a formal possibility, but the fact that our system mimics results obtained with other bone marrow labeling paradigms, including the response to inflammatory injury, argues strongly against this possibility. Furthermore, the occurrence of mononucleated Purkinje neurons, solely because of an unspecific Cre expression would consequentially mean that fusion, or generally transfer of genetic material from hematopoietic cells to other tissues, does not occur at all, neither in healthy nor injured animals. In the light of work done in the field, we find this an unlikely 
option. The fact that we only found one single LacZ-positive PKN containing more than one Y-chromosome argues against the third possibility of stable nuclear fusion. Fusion with subsequent loss of chromosomes from donor cells has been observed in the injured liver after transplantation of human hematopoietic cells (Kashofer et al., 2006). However, we observed only one LacZpositive $\mathrm{PKN}$ with more than one $\mathrm{Y}$ chromosome, suggesting that the loss of chromosomes from fused cells, if it occurs, must be synchronized, very rapid, and efficient. Therefore, we favor the fifth explanation of transient cell fusion or vesicular transport between hematopoietic- and non-hematopoietic cells, enabling the transfer of protein and/or nucleic acid. Release of vesicles containing functional mRNAs from bone marrow derived cells has been reported previously (Valadi et al., 2007) and would be an intriguing way of cell communication between hematopoietic cells and neurons.

In addition to the question of the cell biological mechanisms that regulate endogenous cell fusion, another matter is the role and relevance of this phenomenon. Chromosomal variations of neurons have been described in other contexts (Rehen et al., 2001), and might display, similar to genetic changes because of cell fusion, a contribution to physiological and behavioral variation among individuals not accounted for by classical genetics. As mentioned above, genomic changes caused by potential fusion events might also contribute to pathological processes like tumor formation. However, there is also evidence that BMDCs can contribute to cell maintenance and regeneration of other tissues. Bae et al. (2007) recently reported functional synaptic transmission of PKNs fused with mesenchymal cells from bone marrow. Importantly, we find that inflammation can enhance the contribution of hematopoietic cells to Purkinje neurons. In previous studies, pathological processes were found to increase the contribution of hematopoietic cells to Purkinje neurons liver and muscle (Corbel et al., 2003; Willenbring et al., 2004; Nygren et al., 2008; Johansson et al. 2008). It is important to note that PKNs remained mononucleated in our study despite intrathecal administration of bacterial lipopolysaccharide and increased microglia numbers. This is in contrast to recent findings by Johansson et al. (2008) and Magrassi et al. (2007), who used focal cerebellar and inflammatory injury models and found binucleated PKNs. It suggests that the presence of binucleated PKNs in irradiated bone marrow chimeras, which we confirmed in our study, cannot be explained solely on the basis of irradiation-induced inflammation. Irradiation might impair the ability of cells to extrude genetic material or to influence the association of cells. However, in a recent study, irradiation did not impair the formation of heterokaryons with subsequent loss of chromosomes (Kashofer et al., 2006). In humans, tetraploid PKNs detected by measurement of DNA content, have been described 40 years ago (Lapham, 1968). More recent studies reported the detection of polyploid PKNs in human brain specimens from sex-mismatched bone marrow transplantation recipients (Weimann et al., 2003b). Other groups found these potentially fused cells to be diploid (Cogle et al., 2004), arguing for transdifferentiation. Screening a large number of PKNs from postmortem brains without neuropathological changes and without bone marrow transplantation, we could not confirm the presence of binucleated tetraploid PKNs in the human cerebellum under physiological conditions. This is in line with the results from our mouse model and does not exclude (transient) fusion.

Together, in this study we provide evidence that not only transplanted, but also endogenous BMDCs contribute to different cell types including Purkinje neurons via fusion-like events, and that this process increases in response to inflammation. Further understanding of the underlying mechanisms might help to trigger these potentially regenerative processes and provide, in the long term, new strategies for therapeutical interventions.

\section{References}

Alvarez-Dolado M (2007) Cell fusion: biological perspectives and potential for regenerative medicine. Front Biosci 12:1-12.

Alvarez-Dolado M, Pardal R, Garcia-Verdugo JM, Fike JR, Lee HO, Pfeffer K, Lois C, Morrison SJ, Alvarez-Buylla A (2003) Fusion of bone-marrowderived cells with Purkinje neurons, cardiomyocytes and hepatocytes. Nature 425:968-973.

Bae JS, Han HS, Youn DH, Carter JE, Modo M, Schuchman EH, Jin HK (2007) Bone marrow-derived mesenchymal stem cells promote neuronal networks with functional synaptic transmission after transplantation into mice with neurodegeneration. Stem Cells 25:1307-1316.

Bjerkvig R, Tysnes BB, Aboody KS, Najbauer J, Terzis AJ (2005) Opinion: the origin of the cancer stem cell: current controversies and new insights. Nat Rev Cancer 5:899-904.

Chen EH, Grote E, Mohler W, Vignery A (2007) Cell-cell fusion. FEBS Lett 581:2181-2193.

Cogle CR, Yachnis AT, Laywell ED, Zander DS, Wingard JR, Steindler DA, Scott EW (2004) Bone marrow transdifferentiation in brain after transplantation: a retrospective study. Lancet 363:1432-1437.

Corbel SY, Lee A, Yi L, Duenas J, Brazelton TR, Blau HM, Rossi FM (2003) Contribution of hematopoietic stem cells to skeletal muscle. Nat Med 9:1528-1532.

Crain BJ, Tran SD, Mezey E (2005) Transplanted human bone marrow cells generate new brain cells. J Neurol Sci 233:121-123.

de Boer J, Williams A, Skavdis G, Harker N, Coles M, Tolaini M, Norton T, Williams K, Roderick K, Potocnik AJ, Kioussis D (2003) Transgenic mice with hematopoietic and lymphoid specific expression of Cre. Eur J Immunol 33:314-325.

Georgiades P, Ogilvy S, Duval H, Licence DR, Charnock-Jones DS, Smith SK, Print CG (2002) VavCre transgenic mice: a tool for mutagenesis in hematopoietic and endothelial lineages. Genesis 34:251-256.

Herzog EL, Chai L, Krause DS (2003) Plasticity of marrow-derived stem cells. Blood 102:3483-3493.

Hoffmann O, Priller J, Prozorovski T, Schulze-Topphoff U, Baeva N, Lunemann JD, Aktas O, Mahrhofer C, Stricker S, Zipp F, Weber JR (2007) TRAIL limits excessive host immune responses in bacterial meningitis. J Clin Invest 117:2004-2013.

Horsley V, Pavlath GK (2004) Forming a multinucleated cell: molecules that regulate myoblast fusion. Cells Tissues Organs 176:67-78.

Johansson CB, Youssef S, Koleckar K, Holbrook C, Doyonnas R, Corbel SY, Steinman L, Rossi FM, Blau HM (2008) Extensive fusion of haematopoietic cells with Purkinje neurons in response to chronic inflammation. Nat Cell Biol 10:575-583.

Kashofer K, Siapati EK, Bonnet D (2006) In vivo formation of unstable heterokaryons after liver damage and hematopoietic stem cell/progenitor transplantation. Stem Cells 24:1104-1112.

Krause DS, Theise ND, Collector MI, Henegariu O, Hwang S, Gardner R, Neutzel S, Sharkis SJ (2001) Multi-organ, multi-lineage engraftment by a single bone marrow-derived stem cell. Cell 105:369-377.

Lagasse E, Connors H, Al-Dhalimy M, Reitsma M, Dohse M, Osborne L, Wang X, Finegold M, Weissman IL, Grompe M (2000) Purified hematopoietic stem cells can differentiate into hepatocytes in vivo. Nat Med 6:1229-1234

Lapham LW (1968) Tetraploid DNA content of Purkinje neurons of human cerebellar cortex. Science 159:310-312.

Magrassi L, Grimaldi P, Ibatici A, Corselli M, Ciardelli L, Castello S, Podestà M, Frassoni F, Rossi F (2007) Induction and survival of binucleated Purkinje neurons by selective damage and aging. J Neurosci 27:9885-9892.

Massengale M, Wagers AJ, Vogel H, Weissman IL (2005) Hematopoietic cells maintain hematopoietic fates upon entering the brain. J Exp Med 201:1579-1589.

Mezey E, Key S, Vogelsang G, Szalayova I, Lange GD, Crain B (2003) Transplanted bone marrow generates new neurons in human brains. Proc Natl Acad Sci U S A 100:1364-1369.

Nygren JM, Liuba K, Breitbach M, Stott S, Thorén L, Roell W, Geisen C, Sasse P, Kirik D, Björklund A, Nerlov C, Fleischmann BK, Jovinge S, Jacobsen SE (2008) Myeloid and lymphoid contribution to non-haematopoietic 
lineages through irradiation-induced heterotypic cell fusion. Nat Cell Biol 10:584-592.

Orlic D, Kajstura J, Chimenti S, Jakoniuk I, Anderson SM, Li B, Pickel J, McKay R, Nadal-Ginard B, Bodine DM, Leri A, Anversa P (2001) Bone marrow cells regenerate infarcted myocardium. Nature 410:701-705.

Potgens AJ, Schmitz U, Bose P, Versmold A, Kaufmann P, Frank HG (2002) Mechanisms of syncytial fusion: a review. Placenta 23 [Suppl A]:S107-S113.

Price RW, Brew B, Sidtis J, Rosenblum M, Scheck AC, Cleary P (1988) The brain in AIDS: central nervous system HIV-1 infection and AIDS dementia complex. Science 239:586-592.

Priller J, Persons DA, Klett FF, Kempermann G, Kreutzberg GW, Dirnagl U (2001) Neogenesis of cerebellar Purkinje neurons from gene-marked bone marrow cells in vivo. J Cell Biol 155:733-738.

Rehen SK, McConnell MJ, Kaushal D, Kingsbury MA, Yang AH, Chun J (2001) Chromosomal variation in neurons of the developing and adult mammalian nervous system. Proc Natl Acad Sci U S A 98:13361-13366.

Spees JL, Olson SD, Ylostalo J, Lynch PJ, Smith J, Perry A, Peister A, Wang MY, Prockop DJ (2003) Differentiation, cell fusion, and nuclear fusion during ex vivo repair of epithelium by human adult stem cells from bone marrow stroma. Proc Natl Acad Sci U S A 100:2397-2402.

Stadtfeld M, Graf T (2005) Assessing the role of hematopoietic plasticity for endothelial and hepatocyte development by non-invasive lineage tracing. Development 132:203-213.

Terada N, Hamazaki T, Oka M, Hoki M, Mastalerz DM, Nakano Y, Meyer EM,
Morel L, Petersen BE, Scott EW (2002) Bone marrow cells adopt the phenotype of other cells by spontaneous cell fusion. Nature 416:542-545.

Valadi H, Ekstrom K, Bossios A, Sjöstrand M, Lee JJ, Lötvall JO (2007) Exosome-mediated transfer of mRNAs and microRNAs is a novel mechanism of genetic exchange between cells. Nat Cell Biol 9:654-659.

Wagers AJ, Sherwood RI, Christensen JL, Weissman IL (2002) Little evidence for developmental plasticity of adult hematopoietic stem cells. Science 297:2256-2259.

Wang X, Willenbring H, Akkari Y, Torimaru Y, Foster M, Al-Dhalimy M, Lagasse E, Finegold M, Olson S, Grompe M (2003) Cell fusion is the principal source of bone-marrow-derived hepatocytes. Nature 422:897-901.

Weimann JM, Johansson CB, Trejo A, Blau HM (2003a) Stable reprogrammed heterokaryons form spontaneously in Purkinje neurons after bone marrow transplant. Nat Cell Biol 5:959-966.

Weimann JM, Charlton CA, Brazelton TR, Hackman RC, Blau HM (2003b) Contribution of transplanted bone marrow cells to Purkinje neurons in human adult brains. Proc Natl Acad Sci U S A 100: 2088-2093.

Willenbring H, Bailey AS, Foster M, Akkari Y, Dorrell C, Olson S, Finegold M, Fleming WH, Grompe M (2004) Myelomonocytic cells are sufficient for therapeutic cell fusion in liver. Nat Med 10:744-748.

Wurmser AE, Nakashima K, Summers RG, Toni N, D’Amour KA, Lie DC, Gage FH (2004) Cell fusion-independent differentiation of neural stem cells to the endothelial lineage. Nature 430:350-356.

Ying QL, Nichols J, Evans EP, Smith AG (2002) Changing potency by spontaneous fusion. Nature 416:545-548. 